\title{
Kallistatin inhibits lymphangiogenesis and lymphatic metastasis of gastric cancer by downregulating VEGF-C expression and secretion
}

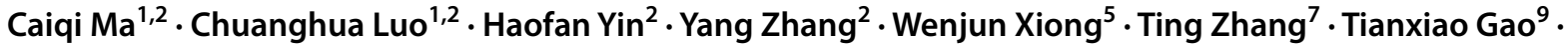

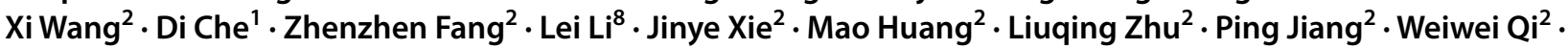

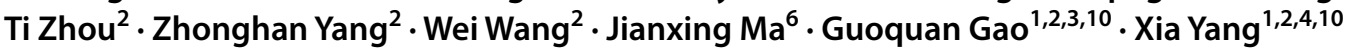

Received: 21 September 2017 / Accepted: 4 December 2017 / Published online: 14 December 2017

(c) The International Gastric Cancer Association and The Japanese Gastric Cancer Association 2017

\begin{abstract}
Background Tumor-induced lymphangiogenesis and lymphatic metastasis are predominant during the metastasis of many types of cancers. However, the endogenous inhibitors that counterbalance the lymphangiogenesis and lymphatic metastasis of tumors have not been well evaluated. Kallistatin has been recognized as an endogenous angiogenesis inhibitor.

Methods and results Our recent study showed for the first time that the lymphatic vessel density (LVD) was reduced in lung and stomach sections from kallistatin-overexpressing transgenic mice. Kallistatin expresses anti-lymphangiogenic activity by inhibiting the proliferation, migration, and tube formation of human lymphatic endothelial cells (hLECs). Therefore, the present study focuses on the relationships of changes in kallistatin expression with the lymphangiogenesis and lymphatic metastasis of gastric cancer and its underlying mechanisms. Our results revealed that the expression of kallistatin in cancer tissues, metastatic lymph nodes, and plasma of gastric cancer patients was significantly downregulated and that the plasma level of kallistatin was negatively associated with the phase of lymph node metastasis. Furthermore, treatment with kallistatin recombinant protein decreased LVD and lymph node metastases in the implanted gastric xenograft tumors of nude mice. Mechanically, kallistatin suppressed the lymphangiogenesis and lymphatic metastasis by downregulating VEGF-C expression and secretion through the LRP6/IKK/IKB/NF-кB signaling pathway in gastric cancer cells.

Conclusions These findings demonstrated that kallistatin functions as an endogenous lymphangiogenesis inhibitor and has an important part in the lymphatic metastasis of gastric cancer.
\end{abstract}

Keywords Kallistatin · Lymphangiogenesis · LRP6 · NF-кB · VEGF-C · Lymph node metastasis · Gastric cancer

Caiqi Ma, Chuanghua Luo, and Haofan Yin contributed equally to this study.

Guoquan Gao

gaogq@mail.sysu.edu.cn

Xia Yang

yangxia@mail.sysu.edu.cn

1 Program of Molecular Medicine, Affiliated Guangzhou Women and Children's Hospital, Zhongshan School of Medicine, Sun Yat-Sen University, Guangzhou, China

2 Department of Biochemistry, Zhongshan School of Medicine, Sun Yat-sen University, Guangzhou 510080, China

3 China Key Laboratory of Tropical Disease Control, Sun Yat-sen University, Ministry of Education, Guangzhou 510080, China

4 Guangdong Engineering \& Technology Research Center for Gene Manipulation and Biomacromolecular Products, Sun Yat-sen University, Guangzhou 510080, China
5 Department of Gastrointestinal Surgery, Traditional Chinese Medicine Hospital of Guangdong Province, Guangzhou, China

6 Department of Physiology, University of Oklahoma, Health Sciences Center, Oklahoma City, OK 73104, USA

7 Department of Clinical Laboratory, Guangzhou First People's Hospital, Guangzhou, China

8 Reproductive Medicine Center, the Third Hospital Affiliated to Guangzhou Medical University, Guangzhou, China

9 Department of Hematologic Oncology, Sun Yat-sen University Cancer Center, Guangzhou 510080, China

10 Department of Biochemistry, Zhongshan Medical School, Sun Yat-sen University, 74 Zhongshan 2nd Road, Guangzhou 510080, China 


\section{Introduction}

Gastric cancer (GC) is one of the most fatal malignancies. It is the fifth leading cause of cancer and the third leading cause of death from cancer, constituting $7 \%$ of cases and $9 \%$ of deaths worldwide. In 2012, gastric cancer occurred in 950,000 people and caused 723,000 deaths [1]. And in 2017, 28,000 new cases and 10,960 new deaths are projected to occur in the United States [2]. The prognosis of gastric cancer is generally poor because metastasis is often discovered late. Among the metastatic paths, lymphatic metastasis is an important one. Thus, investigating novel therapies targeting lymphatic metastasis is an efficient approach in gastric cancer management.

Lymphangiogenesis, the formation of new lymphatics from preexisting lymphatic vasculature by proliferation and migration of lymphatic endothelial cells, is necessary for solid tumor growth and metastasis [3, 4]. The generation of new lymphatic vessels through lymphangiogenesis and the remodeling of existing lymphatics are thought to be important steps in cancer metastasis [5]. The past decade has been exciting in terms of research into the molecular and cellular biology of lymphatic vessels in cancer, and it has been shown that the molecular control of tumor lymphangiogenesis has similarities to that of tumor angiogenesis [6-8]. The process of lymphangiogenesis involves the formation of new lymphatic vessels from preexisting lymphatics, which occurs during embryonic development, wound healing, and in various pathological contexts, including cancer [5]. Vascular endothelial growth factor receptor (VEGFR)-3 is a VEGF receptor, mainly expressed on lymphatic endothelial cells (LEC), which conducts the signals to promote the proliferation and migration of LEC that contribute to the formation of lymphatic tubes [9]. Both VEGF-C and VEGF-D can activate VEGFR-3 to promote the proliferation, migration, and survival of LEC, but VEGF-C shows better efficiency [3, $10,11]$.

Kallistatin (also known as kallikrein-binding protein, $\mathrm{KBP}$ ) is a unique serine proteinase inhibitor (serpin) and a heparin-binding protein. It has been localized in vascular smooth muscle cells and endothelial cells of human blood vessels, suggesting that kallistatin may be involved in the regulation of vascular function [12]. Previous studies have identified it as a novel inhibitor of angiogenesis [13]. Kallistatin also shows a variety of biological effects in pathological and physiological responses, such as blood pressure regulation and anti-inflammation [14-16]. In previous studies, kallistatin markedly inhibited human hepatocellular carcinoma, breast cancer, and colorectal cancer, relying on its anti-angiogenesis activities $[13,17$, 18]. However, the function and the underlying molecular mechanism in tumor-induced lymphangiogenesis have not been determined, so the role of kallistatin in tumorinduced lymphangiogenesis remains to be elucidated.

Our previous work showed that kallistatin possessed anti-lymphangiogenic activity by inhibiting the proliferation, migration, and tube formation of human lymphatic endothelial cells (hLECs) [19]. Therefore, the present study focuses on the relationships of kallistatin expression changes with the lymphangiogenesis and lymphatic metastasis of gastric cancer and its underlying mechanism.

\section{Materials and methods}

\section{Cell lines and cell culture}

HGC-27 and AGS gastric cancer cell lines were purchased from ATCC (American Type Culture Collection; Manassas, VA, USA); MGC-803 and SGC-7901 were purchased from the Cell Bank of China Science Academy (Shanghai, China). The gastric mucosa epithelial cell line GES-1 was purchased from Promocell (Wuhan, China). All these were maintained in Dulbecco's modified Eagle serum (DMEM) supplemented with $10 \%(\mathrm{v} / \mathrm{v})$ fetal bovine serum (FBS), $100 \mathrm{U} / \mathrm{ml}$ penicillin, and $100 \mathrm{mg} / \mathrm{ml}$ streptomycin, then incubated at $37{ }^{\circ} \mathrm{C}$ in a humidified incubator at $5 \% \mathrm{CO}_{2}$.

\section{Tissue specimens and clinicopathological characteristics}

The gastric cancer tissue microarrays (HStm-Ade1201ym-01, HStm-A160CS-02) were purchased from Shanghai Outdo Biotech (Shanghai, China). Samples used in the microarray were histopathologically and clinically diagnosed. The 17 freshly collected gastric cancer and matched adjacent non-tumor gastric tissues from the same patient were frozen and stored in liquid nitrogen until required. The cells were collected and histopathologically and clinically diagnosed at the Traditional Chinese Medicine Hospital of Guangdong Province between 2015 and 2016. Another 11 cases of plasma from gastric cancer patients and all the normal plasmas were collected from the Guangzhou First People's Hospital. The normal plasmas were from the persons who had obtained normal physical examination reports, and the average age of the normal group was controlled similar to the gastric cancer group. Informed consent was obtained from all patients before sample collection, and the use of clinical specimens was also approved by the Institutional Research Ethics Committee. 


\section{Experimental animals}

Twenty BALB/c mice (5-6 weeks old, 18-22 g) were purchased from Vital River Laboratory Animal Technology (Beijing, China). Mice were allowed to acclimate to local conditions for at least 1 week and maintained under a $12 \mathrm{~h}$ dark, $12 \mathrm{~h}$ light cycle with food and water ad libitum. Care, use, and treatment of all animals in the present study were in strict agreement with the institutionally approved protocol according to the United States Public Health Service (USPHS) Guide for the care and use of laboratory animals, as well as the guidelines set forth in the Care and Use of Laboratory Animals by Sun Yat-sen University. The animal use protocol has been reviewed and approved by the institutional animal care and use committee of Sun Yat-sen University (IACUC SYSU, No. 20061211005).

\section{Popliteal lymph node metastasis model}

The gastric cancer cell line HGC-27 $\left(2 \times 10^{6}\right)$ previously infected by pLenti-CMV-EGFP-linker-Luc-PGK-Puro (OBiO Biotech, Shanghai, China) was inoculated into the foot-pads of the two groups of mice (each containing 10 mice) on day 0 . When the tumors in the foot-pads reached a volume of $\sim 100 \mathrm{~mm}^{3}$, they were imaged with the IVIS Spectrum Imaging System (Perkin Elmer) after injection of luciferin (Promega), and mice were then randomized into two groups. The rKAL-treated group received an intraperitoneal injection of recombinant kallistatin at 48-h intervals to a total amount of $2.88 \mathrm{mg} / \mathrm{kg}$ body mass $\mathrm{rKAL}$; the control group was treated with the same volume of phosphate-buffered saline. Tumor growth was monitored by external measurement in two dimensions. Tumor volume was calculated by the following formula: volume $\left(\mathrm{mm}^{3}\right)=\left(\right.$ length $\times$ width $\left.^{2}\right) / 2$. 28 days after the first injection, tumors in the foot-pads and popliteal lymph nodes were imaged with the IVIS Spectrum Imaging System. The mice were then killed and tumors were dissected and weighed. Last, tumors and popliteal lymph nodes were enucleated and embedded in paraffin. Serial $5-\mu \mathrm{m}$ sections were analyzed by green fluorescent protein (GFP) imaging and immunohistochemistry (IHC) with antiLYVE-1 antibodies (Abcam, Cambridge, MA, USA). The images were captured using an AxioVision Rel.4.6 system (Carl Zeiss, Jena, Germany).

\section{Expression and purification of recombinant kallistatin}

The recombinant kallistatin (rKAL) cDNA containing a sequence encoding the full-length mature peptide was amplified from the total RNA of rat liver by reverse transcriptionpolymerase chain reaction (PCR). The PCR product was cloned into the pET28 vector (Novagen) at the BamHI and
SacI sites in frame with the sequence encoding the His tag at the 3 '-end. The kallistatin/pET28 construct was introduced into Escherichia coli strain BL-21/DE3 (Novagen). The expression of kallistatin was induced by the addition of isopropylthio- $\beta$-galactoside (IPTG) and carried out for $10 \mathrm{~h}$ at $25^{\circ} \mathrm{C}$. Periplasmic proteins were released by breaking down bacteria ultrasonically and separated from cells by centrifugation. Kallistatin purification and lipopolysaccharide (LPS) deletion were accomplished by dialysis with $1 \mathrm{~K}$ MWCO (molecular weight cutoff) dialysis membranes, and LPS level was detected in the allowed scope. The identity of recombinant kallistatin was examined by sodium dodecyl sulfate-polyacrylamide gel electrophoresis (SDS-PAGE) and Western blot analysis using antibody specific to His-tag. Last, the concentration of recombinant kallistatin was measured by bicinchoninic acid assay (BCA) assay, and bacteria were eliminated with a $0.22-\mu \mathrm{m}$ filter. An average of $20 \mathrm{mg}$ purified kallistatin in soluble form was obtained from 11 of culture.

\section{Enzyme-linked immunosorbent assay (ELISA)}

The freshly collected plasma, gastric cancer, and matched adjacent non-tumor gastric tissues from the same patient were frozen and stored in liquid nitrogen until required. They were collected and histopathologically and clinically diagnosed at the Traditional Chinese Medicine Hospital of Guangdong Province between 2015 and 2016. The gastric cancer cells seeded in 6-well culture plates were cultured in the growth medium until $90 \%$ confluence. The cells were washed three times with phosphate-buffered saline (PBS) and the growth medium was replaced by DMEM; kallistatin $(640 \mathrm{nM})$ was then added to the medium and incubated with the cells for the time indicated. PBS was added as control. Finally, kallistatin or VEGF-C in plasma, homogenate of fresh tissues, and conditioned media was determined using human kallistatin (Catalog Number: DY1669; R\&D Systems, USA) and VEGF-C (Catalog Number: DVEC00; R\&D Systems, USA) ELISA kits.

\section{Hematoxylin and eosin (H\&E) staining and immunohistochemistry}

Tissues were surgically resected, fixed in formalin, and embedded in paraffin. Then, 5 - $\mu \mathrm{m}$-thick histological sections were prepared. Before immunohistochemistry, histological sections were stained with hematoxylin and eosin (H\&E) to observe the morphology. The sections were treated with endogenous peroxidase blocking solution and normal goat serum to block nonspecific background. Sections were then incubated with antibodies: kallistatin, D2-40, VEGF-C (Abcam, Cambridge, MA, USA). After overnight incubation at $4{ }^{\circ} \mathrm{C}$, sections were incubated with a biotin-conjugated 
second antibody at room temperature for $20 \mathrm{~min}$ and incubated with enzyme conjugate [horseradish peroxidase (HRP)-streptavidin] under the same conditions. The vessels were revealed with streptavidin-peroxidase followed by chromogenic substrate diaminobenzidine (DAB), and the sections were counterstained with hematoxylin. Images of tissue microarrays were taken by Pannoramic Viewer software, and quantification of expression level was determined by gray-scale scanning with Image $\mathbf{J}$ software. Lymphatic vessel density (LVD) was determined by the tube number per $100 \times$ field.

\section{Cytoplasmic and nuclear protein extraction}

After incubation with $640 \mathrm{nM} \mathrm{rKAL}$ or PBS for $24 \mathrm{~h}$, HGC27 cells were harvested. The cytoplasmic and nuclear protein were extracted with an extraction kit (Thermo) according to its protocol. Then, P65, histone, and $\beta$-actin in nuclear protein were detected by Western blot.

\section{Western blotting}

Kallistatin antibody was purchased from Abcam (Cambridge, MA, USA) and LRP6 from Santa Cruz Biotechnology (Santa Cruz, CA, USA). IKK, p-IKK, IкB, p-IкB, $\mathrm{P} 65$, p-P65, VEGF-C, histone, and $\beta$-actin antibodies were purchased from CST (Danvers, MA, USA). Cells were harvested and lysed for total protein extraction. Protein concentration was determined using a BCA protein assay kit (Keygen, Nanjing, China) according to the manufacturer's protocol. Aliquots of equal amounts of protein from the cell lysate were subjected to Western blot analysis. Densitometry was performed using ImageJ software and normalized by $\beta$-actin levels.

\section{siRNA transfection}

LRP6 siRNA and a nonspecific siRNA (control) were purchased from RiboBio (Guangzhou, Guangdong, China). According to the manufacturer's instructions, transfections were performed at approximately $60 \%$ confluency using Lipofectamine 2000 (Invitrogen, Carlsbad, CA, USA). For each transfection reaction, $20 \mathrm{nM}$ LRP6 siRNA or control siRNA was used for preparation of siRNA-transfection complexes at room temperature for $20 \mathrm{~min}$. Transfections were performed in $0.5 \mathrm{ml}$ (12-well plate) or $1.5 \mathrm{ml}$ (6-well plate) serum-free medium for $8 \mathrm{~h}$. After incubation, transfection complexes were removed and replaced with their corresponding media. Transfection efficiency (80-90\%) was determined by Western blot analysis. Cells were utilized for other experiments at 24-72 $\mathrm{h}$ after transfection.

\section{Quantitative (q)RT-PCR}

Total RNA was extracted from gastric cancer cell lines according to the manufacturer's instructions for the kit (Tiangen, Beijing, China). Of the total RNA, $500 \mathrm{ng}$ was used for reverse transcription using a PrimeScript1RT reagent Kit Perfect Real Time kit (Takara Bio, Shiga, Japan), and then subjected to quantitative real-time PCR analysis (qRT-PCR) using SYBR1 Premix Ex TaqTM (Perfect Real Time) (Takara Bio) and a Roche's capillary-based LightCycler1 2.0 Systems (Roche Diagnostics, Indianapolis, IN, USA). Human cDNA was amplified with specific primers for human LRP6 (sense primer: 5'-TTTGGATGGGACAGA ACGGG-3', antisense primer: 5'-TCCGGTTAGCACCTG AGAGA-3'), human VEGF-C (sense primer: 5'-CTCAAG GCCCCAAACCAGTA-3', antisense primer: 5'-GCCTGA CACTGTGGTAGTGTT-3') $\beta$-actin (sense primer: $5^{\prime}$-ACT CTTCCAGCCTTCCTTC-3', antisense primer: 5'-ATCTCC TTCTGCATCCTGTC-3') (GenRay, Guangzhou, China). Target mRNA was determined using the comparative cycle threshold method of relative quantitation. The calibrator sample was isolated from untreated gastric cancer cells, with $\beta$-actin used as an internal control. All samples were assayed in triplicate.

\section{Immunofluorescence}

Gastric cancer cells were plated on culture slides, and incubated with PBS or kallistatin $(640 \mathrm{nM})$ for $6 \mathrm{~h}$. The cells were washed and fixed in $4 \%$ paraformaldehyde. Then, cells were blocked with normal nonimmune goat serum at $37{ }^{\circ} \mathrm{C}$ for $1 \mathrm{~h}$. After washing three times, the cells were incubated with rabbit phospho-P65 and P65 antibodies (Abcam, Cambridge, MA, USA) at $37^{\circ} \mathrm{C}$ for $2 \mathrm{~h}$, then incubated with fluorescein isothiocyanate (FITC)-conjugated goat antirabbit IgG (Dako, Glostrup, Denmark) at $37^{\circ} \mathrm{C}$ for $1 \mathrm{~h}$ after three washings. Finally, the cell nucleus was stained with 4,6-diamino-2-phenylindole (DAPI) (Sigma-Aldrich, St. Louis, MO, USA). Cells were visualized under a confocal microscope (Carl Zeiss, Oberkochen, Germany). In negative-control staining, the primary antibodies were omitted.

\section{Co-immunoprecipitation (Co-IP) assay}

Cells were washed with ice-cold PBS and lysed for $20 \mathrm{~min}$ on ice in RIPA lysis buffer (Applygen, Beijing, China) supplemented with protease inhibitor cocktail (Sigma-Aldrich) and phenylmethylsulfonyl fluoride (PMSF) (Sigma-Aldrich). Lysates were pre-cleared then incubated overnight at $4{ }^{\circ} \mathrm{C}$ with the indicated antibody and protein A-Sepharose CL-4B beads ( $500 \mu \mathrm{g}$ protein; $5 \mu \mathrm{g} \mathrm{IgG} ; 50 \mu \mathrm{l}$ beads; Invitrogen, Carlsbad, CA, USA). Immunoprecipitates were washed twice with RIPA lysis buffer. Proteins were eluted from 
beads with SDS sample buffer and then subjected to Western blotting analysis.

\section{Chromatin immunoprecipitation assay}

Cells $\left(2 \times 10^{6}\right)$ plated in a 100 -mm culture dish were treated with $1 \%$ formaldehyde to cross-link proteins to DNA. The cell lysates were sonicated to shear the DNA into 300- to 1000-bp lengths. Aliquots containing equal amounts of chromatin supernatants were incubated on a rocking bed at $4{ }^{\circ} \mathrm{C}$ overnight with either $1 \mu \mathrm{g}$ anti-P65 antibody or $1 \mu \mathrm{g}$ anti-IgG antibody as a negative control. Following reverse cross-linking of protein-DNA complexes to free the DNA, real-time PCR was carried out. The primers used in this study are listed in the online supplementary materials and methods.

\section{Luciferase assay}

HGC-27 cells were seeded in 24-well plates $24 \mathrm{~h}$ before transfection, then transiently co-transfected with VEGF-CLuc (this vector contains multiple copies of VEGF-C element fused to the HSV-TK promoter, a gift from Professor Jun Li from Sun Yat-sen University) and pRL-TK Renilla luciferase plasmids by using Attractene Transfection Reagent (Qiagen, Duesseldorf, Germany). A negative-control vector pGL3-basic was added at identical DNA to subtract the background fluorescence. Forty-eight hours after transfection, cells were pre-stimulated with kallistatin $(640 \mathrm{nM})$ or PBS for $6 \mathrm{~h}$. Cells were then lysed using $100 \mathrm{ml}$ passive lysis buffer (Promega, Madison, WI, USA), and the firefly luciferase (VEGF-C-Luc) activity and Renilla luciferase activity were measured by using the Dual-Luciferase Reporter system (Promega) according to user's manual in Luminometer (Berthold Centro LB 960, Germany), and normalized by Renilla luciferase activity.

\section{Statistical analysis}

Data are presented as mean $\pm \mathrm{SD}$. Comparisons were performed by two-tailed paired Student's $t$ test or one-way analysis of variance (ANOVA). $p<0.05$ was considered statistically significant.

\section{Results}

\section{Kallistatin was reduced in the cancer tissue and plasma of gastric cancer patients}

Kallistatin was reported as a potent inhibitor of angiogenesis, and it also demonstrates effective in inhibiting cancer cells. Sun et al. found that the mRNA and protein expression of kallistatin in colorectal cancer (CRC) specimens was significantly lower than that in adjacent normal mucosa [20]. Here we applied a tissue microarray (HStmAde120lym-01) to detect the kallistatin level in gastric cancer, and the immunochemistry results showed kallistatin was obviously lower in gastric cancer tissues than in the adjacent normal tissues (Fig. 1a, b, c). The ELISA kit was then applied to test tissue kallistatin in gastric cancer and adjacent normal tissues. Finally, similar results with IHC were obtained (Fig. 1d). Next, fresh plasma was collected from 32 normal persons and 28 gastric cancer patients to determine the concentration of kallistatin. Figure 1e shows that plasma kallistatin in gastric cancer patients was nearly twofold lower than in normal persons. These findings suggest that kallistatin is likely to be involved in the progress of gastric cancer.

To confirm the correlation between kallistatin expression and clinical features, we ordered another tissue microarray with detailed clinical features that contains 80 cancerous and adjacent tissues (\#HStmA160CS02) and then analyzed the correlation between kallistatin expression and clinical features. Interestingly, the decrease of kallistatin is correlated with gastric cancer lymph node metastasis ( $p<0.05$, Table 1$)$, but no correlation occurred with other clinical features.

\section{Kallistatin was related to the lymph node metastasis of gastric cancer}

Our findings had previously demonstrated that kallistatin inhibited the growth of gastric cancer by reducing vascular endothelial growth factor production and angiogenesis [21]. Considering that lymph node metastasis has an important role in the process of gastric cancer metastasis and lymphangiogenesis shares a similar mechanism with angiogenesis [6], it is necessary to determine the effect of kallistatin on lymph node metastasis of gastric cancer cells. First, we analyzed the relationship between plasma kallistatin and the phase of lymph node metastasis (Fig. 1f): a negative correlation was found, which suggested that kallistatin was a potential inhibitor of lymph node metastasis. Lymphangiogenesis is an important step in the process of lymph node metastasis, so the lymphatic marker D2-40 was employed to stain the lymphatic tubes, and then the lymphangiogenesis indicator lymphatic vessel density (LVD) was analyzed. At last, the outcome showed that LVD in gastric cancer areas where less kallistatin was seen was significantly higher than in the normal adjacent areas (Fig. 1g, h). These results indicate that lower kallistatin expression may be a significant factor in promoting the lymph node metastasis of gastric cancer. 
a

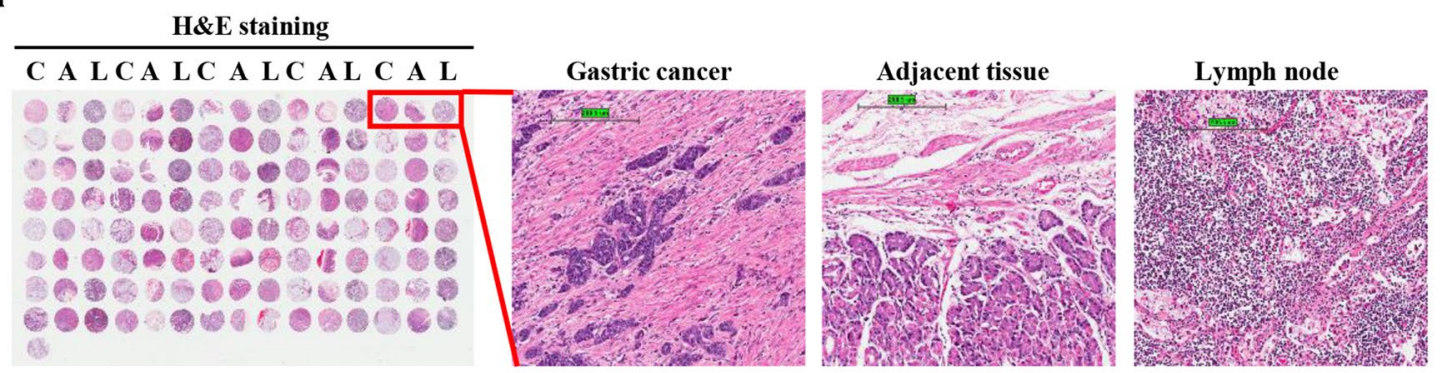

b

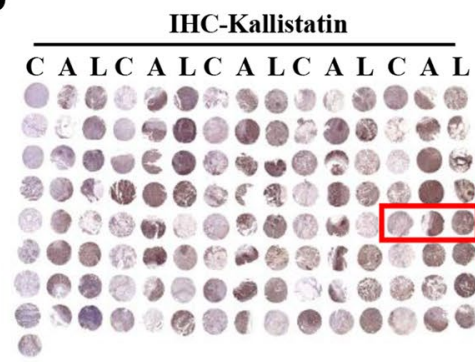

C

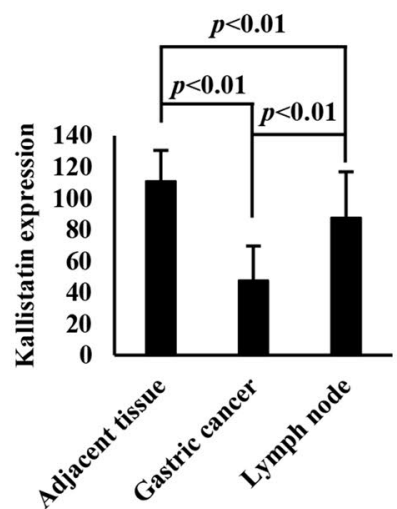

$\mathbf{f}$

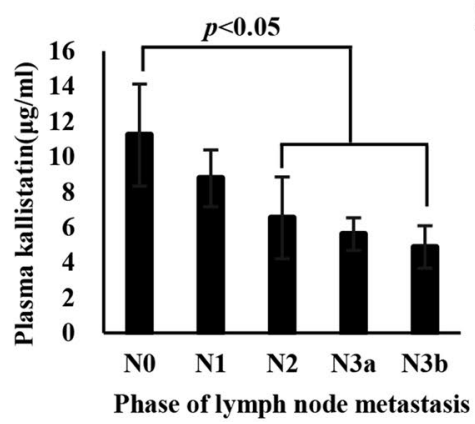

Gastric cancer

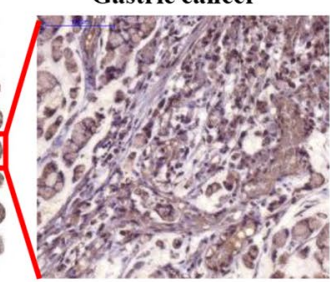

Adjacent tissue

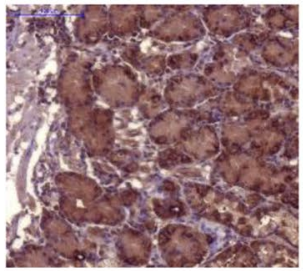

Lymph node

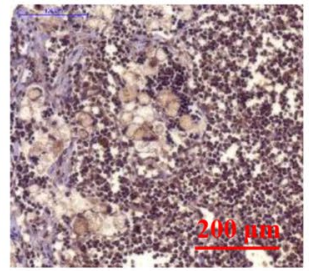

d

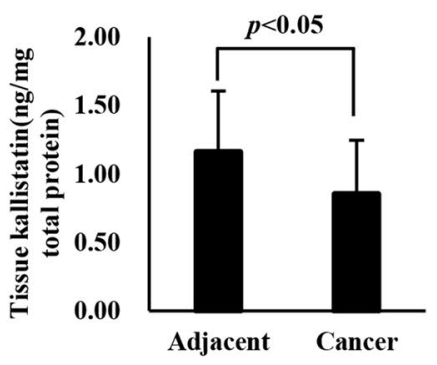

g

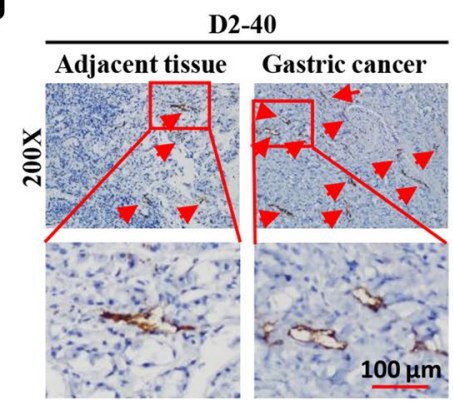

e

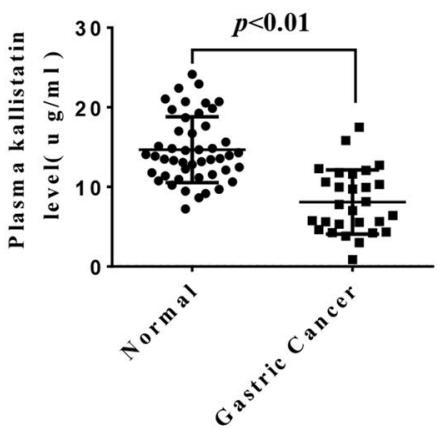

h

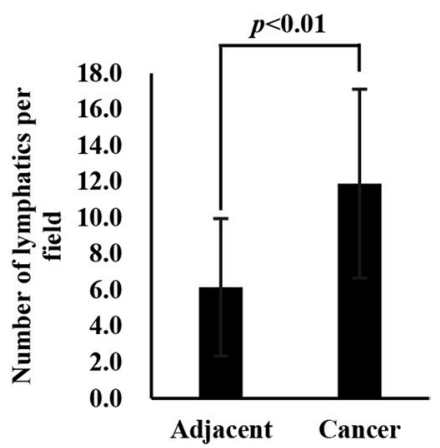

Fig. 1 Lower level of kallistatin in tumor tissue and plasma of gastric cancer patients was correlated with lymph node metastasis. a Hematoxylin and eosin (H\&E) staining of tissue microarray, which contains 40 gastric cancer tissues $(C), 40$ adjacent tissues $(A)$, and 40 lymph nodes (L). Bar $200 \mu \mathrm{m}$. b Kallistatin staining of tissue microarray by immunochemistry (IHC) (tissue microarray is from the same samples just described). Bar $200 \mu \mathrm{m}$. c Kallistatin expression of different tissues in the microarray quantified by gray-scale scanning with Image J. d Tissue kallistatin level of adjacent tissues (17) and gas- tric cancer tissues (17) detected by enzyme-linked immunosorbent assay (ELISA) kit. e Comparison of average plasma kallistatin level from 32 normal people and 28 gastric cancer patients. f Relationship between plasma kallistatin and phase of lymph node metastasis for samples N0 to N3b (5, 4, 4, 4, 5, respectively. g, h Lymphatic vessel density (LVD) in gastric cancer tissues and adjacent tissues: lymphatic tubes were stained with D2-40. Histogram represents LVD in adjacent tissues (17) and gastric cancer tissues (17). Results are mean $\pm \mathrm{SD}$. Statistical significance determined using $t$ test 
Table 1 Correlation between kallistatin expression and clinical features

\begin{tabular}{|c|c|c|c|c|}
\hline \multirow[t]{2}{*}{ Characteristic } & \multirow[t]{2}{*}{ All cases } & \multicolumn{2}{|c|}{$\begin{array}{l}\text { Expression of } \\
\text { kallistatin }\end{array}$} & \multirow[t]{2}{*}{$P$ value } \\
\hline & & Low & High & \\
\hline Sex & & & & 0.353 \\
\hline Female & 18 & 13 & 5 & \\
\hline Male & 62 & 35 & 27 & \\
\hline Age (years) & & & & 0.819 \\
\hline$>63.8$ & 40 & 25 & 15 & \\
\hline$\leq 63.8$ & 40 & 23 & 17 & \\
\hline Clinical stage & & & & 0.141 \\
\hline I-II & 51 & 27 & 24 & \\
\hline III-IV & 29 & 21 & 8 & \\
\hline $\mathrm{T}$ classification & & & & 0.323 \\
\hline $\mathrm{T} 1-\mathrm{T} 2$ & 8 & 3 & 5 & \\
\hline T3-T4 & 72 & 45 & 27 & \\
\hline $\mathrm{N}$ classification & & & & 0.036 \\
\hline N0 & 17 & 6 & 11 & \\
\hline N1 & 14 & 7 & 7 & \\
\hline N2 & 22 & 14 & 8 & \\
\hline $\mathrm{N} 3$ & 27 & 21 & 6 & \\
\hline M classification & & & & 0.294 \\
\hline M0 & 73 & 42 & 31 & \\
\hline M1 & 7 & 6 & 1 & \\
\hline Differentiation & & & & 0.678 \\
\hline Poorly & 34 & 19 & 15 & \\
\hline Moderately, highly & 46 & 29 & 17 & \\
\hline
\end{tabular}

The tissue microarray containing 80 pairs of tissues was obtained from Shanghai Outdo Biotech (\#HStmA160CS02, Shanghai, China). Kallistatin was detected by immunochemistry (IHC); then, every section was evaluated and scored independently by two pathologists. A semiquantitative scoring system was used in this trial (Sinicrope FA et al., Cancer Research, 1995; Li Q et al., American Journal of Cancer Research, 2015). The percentage of positively stained cells was scored as $0,<5 \% ; 1,5-25 \% ; 2,26-50 \% ; 3,51-75 \% ; 4,>75 \%$. Intensity was scored as "-" (negative), "+" (weak), "++" (moderate), or "+++" (strong). We multiplied the percentage score by the staining intensity score to generate the immunoreactivity score (IS). High expression of kallistatin was defined as detectable immunoreactions in cytoplasm and unclear with IS $\geq 4$. Statistical significance was determined by chi-square test

\section{Kallistatin inhibited the growth and lymphatic metastasis of gastric cancer in vivo}

To investigate the direct effect of kallistatin on lymph node metastasis in vivo, the popliteal lymph node metastasis model was applied. After 28 days treatment with $640 \mathrm{nM}$ rKAL by intraperitoneal injection, HGC-27-grafted gastric cancer in the foot-pads and the popliteal lymph nodes in the rKAL-treated mice was both smaller and lighter than in their counterparts (Fig. $2 a-c, h-j$ ). Among them,
Fig. $2 \mathrm{~h}$ showed the potent inhibitory effect of kallistatin on lymph node metastasis. The LVD in HGC-27-grafted gastric cancer treated with rKAL was also lower than in the control group (Fig. 2f, g). To confirm the enlarged lymph nodes were related to the metastasized gastric cancer cells rather than the tumor-induced inflammation, we labeled the HGC-27 cells with GFP and luciferase previously, then detected the gastric cancer cells that had metastasized to the popliteal lymph nodes by live imaging with luciferin. After dissecting the popliteal lymph nodes, the GFP-labeled gastric cancer cells were observed with the fluorescence microscope. The metastasized cancer cells in the popliteal lymph nodes from the rKAL-treated group were much fewer than those in the control group Fig. 2d, e). These results confirm the inhibitory effect of kallistatin on lymph node metastasis in gastric cancer.

\section{Expression and secretion of kallistatin and VEGF-C were negatively correlative}

The preceding work has verified the inhibitory effect of kallistatin on lymph node metastasis. However, the underlying mechanism is still unknown. As we described previously, VEGF-C is a predominant vascular endothelial growth factor that promotes lymphangiogenesis and lymph node metastasis. And, our previous study revealed that kallistatin had no effect on the proliferation and migration of gastric cancer cells [21]. To clarify the mechanism of kallistatin inhibition of the lymph node metastasis of gastric cancer, the influence of kallistatin on expression and secretion of VEGF-C in gastric cancer cells was investigated. First, we detected the plasma VEGF-C in gastric cancer patients and tissue VEGF-C in gastric cancer, finding that both of these were higher than their normal counterparts (Fig. 3a, b, c). The relationship between plasma kallistatin and VEGF-C in gastric cancer patients was then analyzed, showing that these were negatively correlated (Fig. 3d; $r=-0.51, p=0.036$ ). Moreover, the expression of kallistatin and VEGF-C in gastric cancer lines in different degrees of differentiation was examined, showing that the lower the degree of gastric cancer cell differentiation, the less kallistatin and the more VEGF-C expressed (Fig. 3e). To confirm the correlation between cell differentiation and lymph node metastasis in vivo, a tissue microarray with detailed clinical features that contains 80 cancerous and adjacent tissues was analyzed. Interestingly, the results showed the decrease of kallistatin is correlated with gastric cancer lymph node metastasis $(p<0.05$, Table 1$)$, but no correlation was found between the differentiation of gastric cancer cells and lymph node metastasis (Table 1). 
a

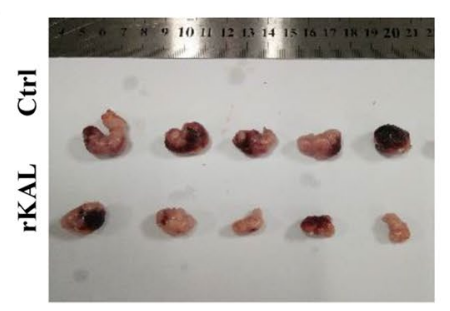

b

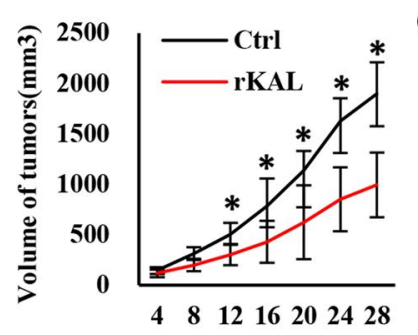

C

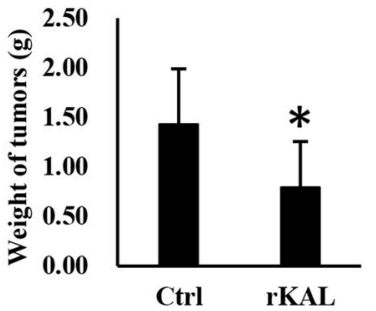

d

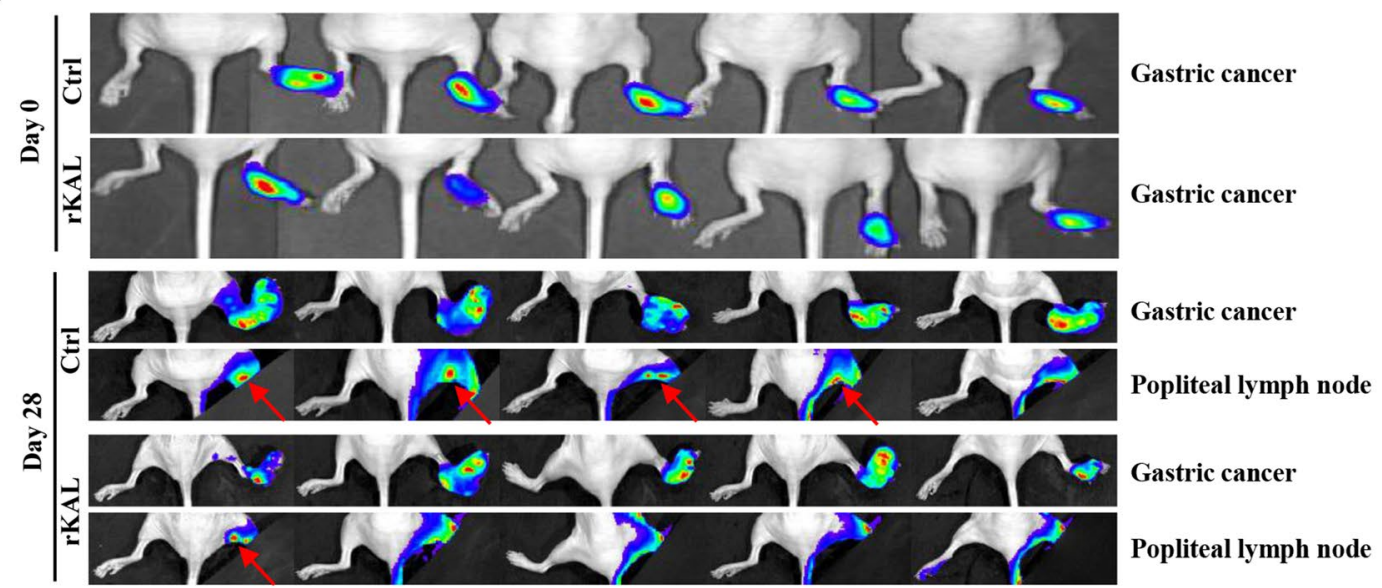

e
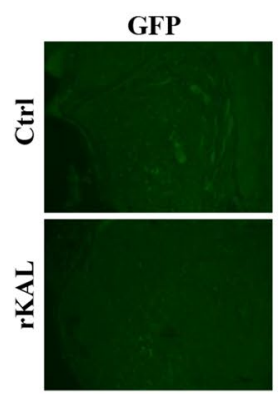

h
DAPI
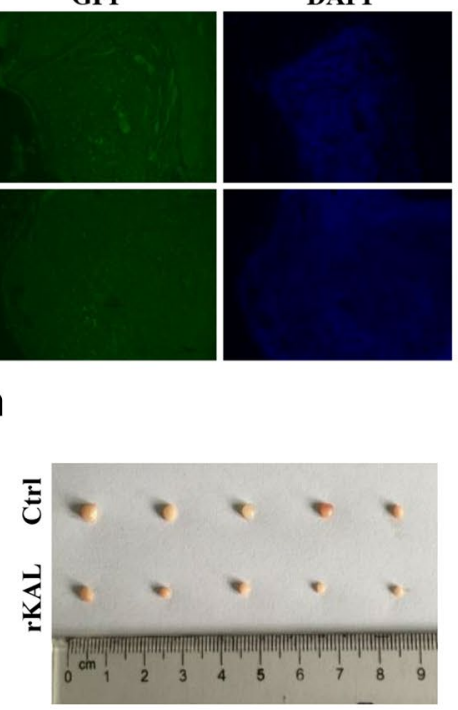

f
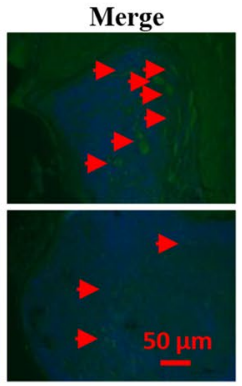

i

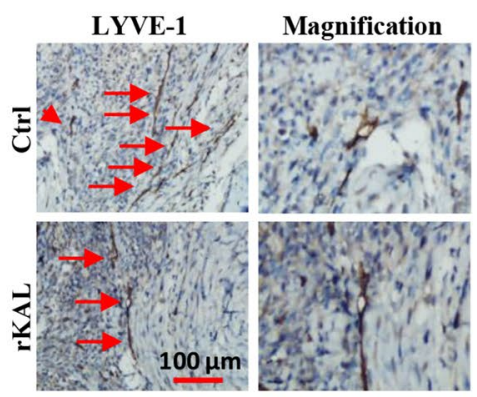

g
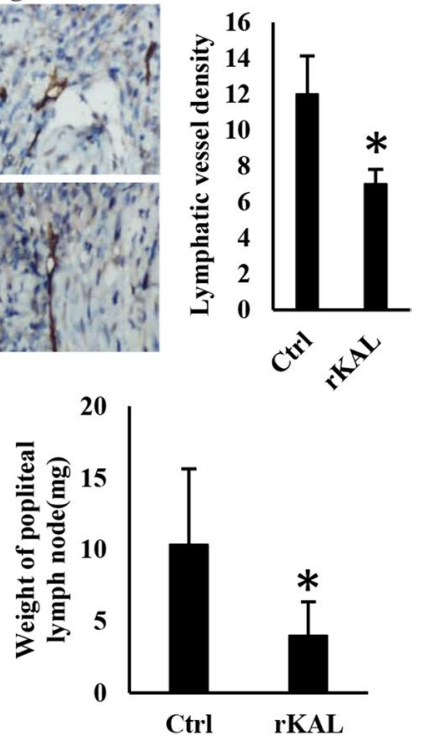

Fig. 2 Kallistatin inhibited gastric cancer growth and its lymph node metastasis in vivo. a, b, c Foot-pad gastric tumors from nude mice treated with phosphate-buffered saline (PBS) or recombinant kallistatin (rKAL), respectively, for 28 days; histogram represents tumor volume and weight of tumors. d Gastric cancer cells that had metastasized to the homolateral popliteal lymph nodes were imaged by live imaging with the IVIS system after luciferin injection; red arrows show the metastasized gastric cancer cells previously labeled with luciferase. e Gastric cancer cells that had metastasized to the homolateral popliteal lymph nodes were imaged by fluorescence micro- scope; red arrows show the metastasized gastric cancer cells labeled with green fluorescent protein (GFP) beforehand. f, $\mathbf{g}$ Lymphatic vessel density (LVD) in foot-pad gastric tumors with lymphatic tubes stained with LYVE-1; histogram represents LVD from rKAL group (5) and PBS group (5). LVD was determined by tube number per $100 \times$ field. h, i, j Popliteal lymph nodes from animal models after treatment with PBS or rKAL for 28 days; histogram represents volume and weight of each group of lymph nodes. Statistical significance determined using $t$ test at $* p<0.05$, $* * p<0.01$. Results are means $\pm \mathrm{SD}$ 
a

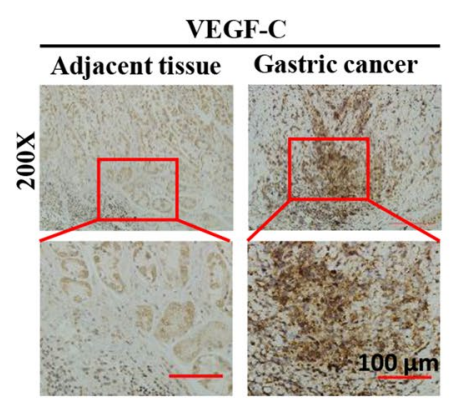

b

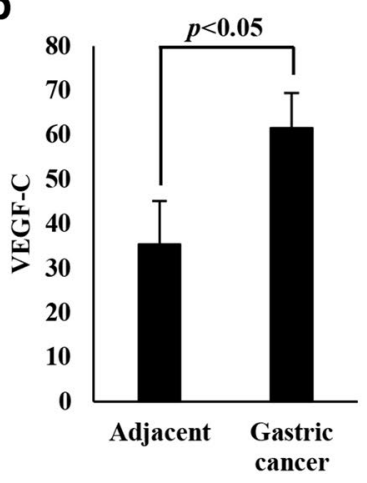

C

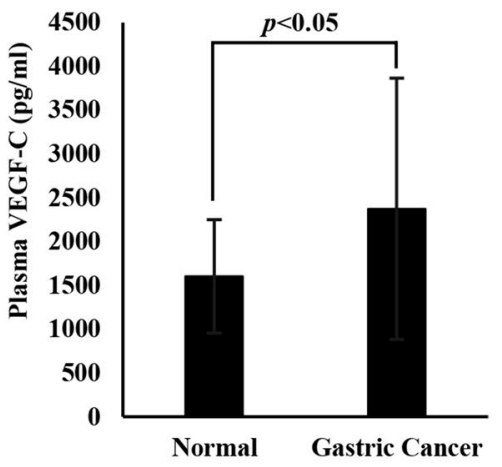

d

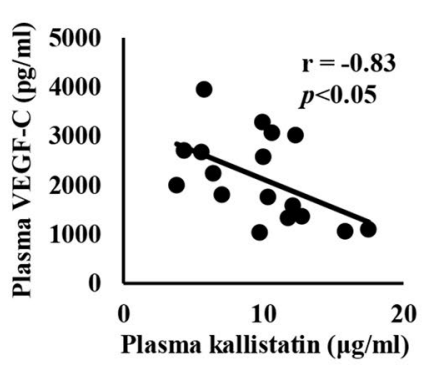

e

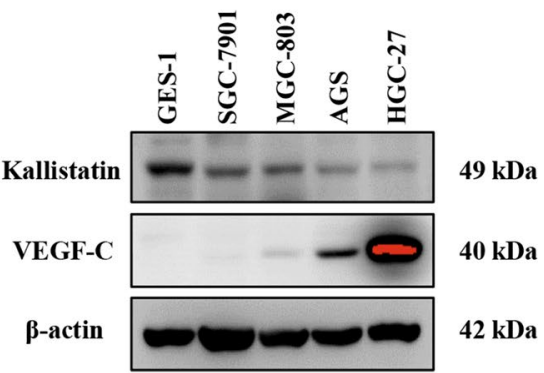

Fig. 3 Lower kallistatin level was correlated with elevated vascular endothelial growth factor (VEGF)-C level in gastric cancer cells. a, b Expression of VEGF-C in gastric cancer tissues and adjacent tissues detected by immunochemistry (IHC); histogram represents expression level of VEGF-C quantified by gray-scale scanning with Image J. c Plasma VEGF-C level in 32 normal persons and 28 gastric cancer patients. Results are mean \pm SD. d Concentration rela-

\section{Kallistatin downregulated the expression and secretion of VEGF-C in gastric cancer cells}

To further investigate the relationship between kallistatin and VEGF-C in gastric cancer cells, we performed a series of in vitro studies. First, the VEGF-C mRNA level in HGC-27 was measured, and the data showed that kallistatin reduced the transcription of VEGF-C (Fig. 4a). A similar outcome was obtained by dual-luciferase reporter assay (Fig. 4b). Then, the Western blot results showed that recombinant kallistatin treatment inhibited the VEGF-C expression of gastric cancer cells in a dose- and timedependent manner (Fig. 4e, f). Moreover, kallistatin overexpression by adenovirus also downregulated the expression level of VEGF-C (Fig. 4g, h). The secretion level of VEGF-C was detected with a specific ELISA kit, and the results showed that secretion of VEGF-C was also inhibited by kallistatin (Fig. 4c, d). Last, the VEGF-C level in gastric cancer cells from foot-pad tumors was measured by immunochemistry, which finally verified that VEGF-C expression in gastric cancer cells was reduced by recombinant tionship between plasma kallistatin and plasma VEGF-C from 17 gastric cancer patients. Statistical significance determined by Pearson's correlation test at $p<0.05$. e Expression level of kallistatin and VEGF-C in gastric mucosal epithelial cells and different gastric cancer cell lines in different degrees of differentiation. Statistical significance was determined with $t$ test. $* p<0.05, * * p 0.01$. Results are means $\pm \mathrm{SD}$

kallistatin (Fig. 4i, j). Taken together, our results showed that kallistatin could downregulate the expression and secretion of VEGF-C in gastric cancer cells.

\section{The NF- $\mathrm{B}$ pathway is involved in the downregulation of VEGF-C expression by kallistatin}

It was previously reported that the NF-кB signaling pathway was involved in the regulation of VEGF-C expression [22] and that kallistatin could inhibit the activation of NF-кB signaling [23]. Here we tested whether kallistatin regulates VEGF-C expression through inhibiting NF- $\mathrm{KB}$ activation. Figure 5a, b demonstrates that NF-кB P65 acted as the transcript factor of VEGF-C in gastric cancer cells, which indicated that NF- $\mathrm{KB}$ participated in the regulation of VEGF-C expression. Then, the activity of kallistatin on NF-кB signaling was detected, showing that both the activation of NF- $\mathrm{KB}$ and the translocation of P65 were inhibited by kallistatin (Fig. 5c, d, e). Next, the NF-кB activator IL-1 $\beta(0.1 \mathrm{ng} / \mathrm{ml})$ was applied to rescue 


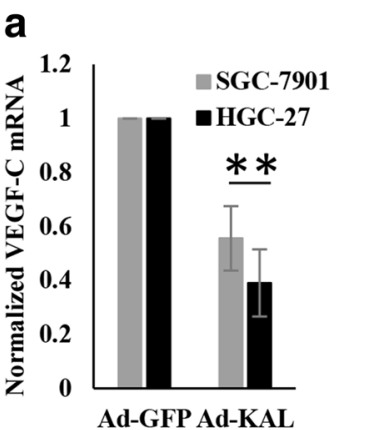

b

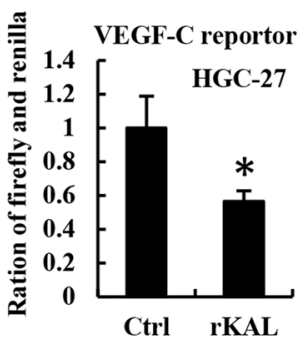

e

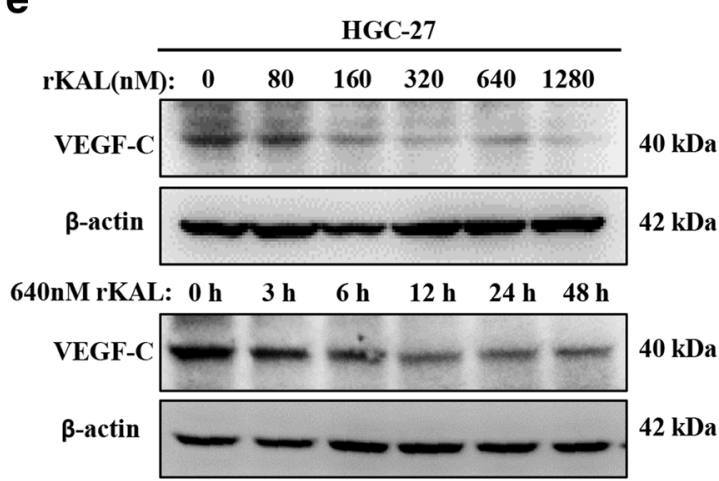

g

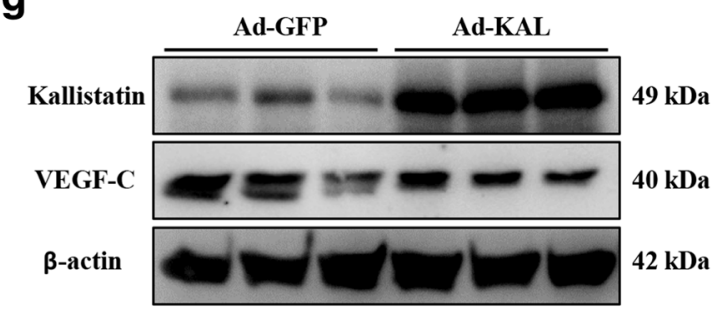

i

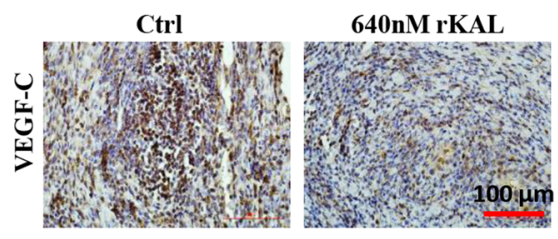

Fig. 4 Kallistatin inhibited the expression and secretion of VEGF-C. a VEGF-C mRNA level in HGC-27 treated by Ad-KAL or Ad-GFP, measured by qRT-PCR in three independent experiments. b VEGF$\mathrm{C}$ transcription level in HGC-27 treated by Ad-KAL or Ad-GFP detected by dual-luciferase reporter assay. $\mathbf{c}, \mathbf{d}$ Effect of kallistatin on secretion of VEGF-C by gastric cancer cells HGC-27 and SGC-7901; supernatant VEGF-C was detected by an ELISA kit. e, f Kallistatin dose- and time-dependently downregulated VEGF-C expression in gastric cancer cells. $\mathbf{g}$, h Overexpression of kallistatin by adenovirus

the inhibition of kallistatin, and in the end, the expression and secretion of VEGF-C were recovered (Fig. 5f, g). These findings suggest that kallistatin downregulated VEGF-C expression through inhibiting the NF-кB

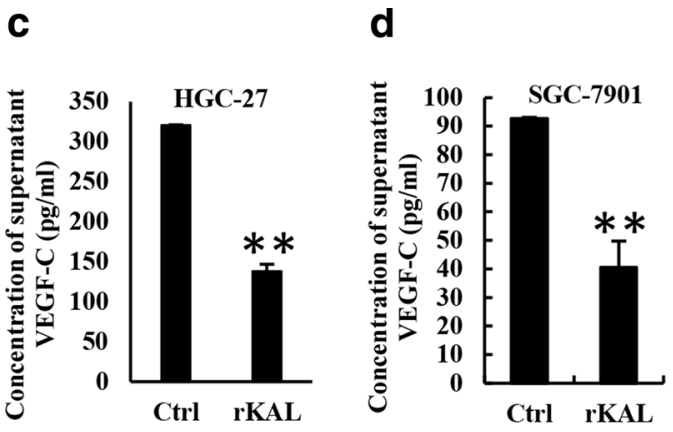

f

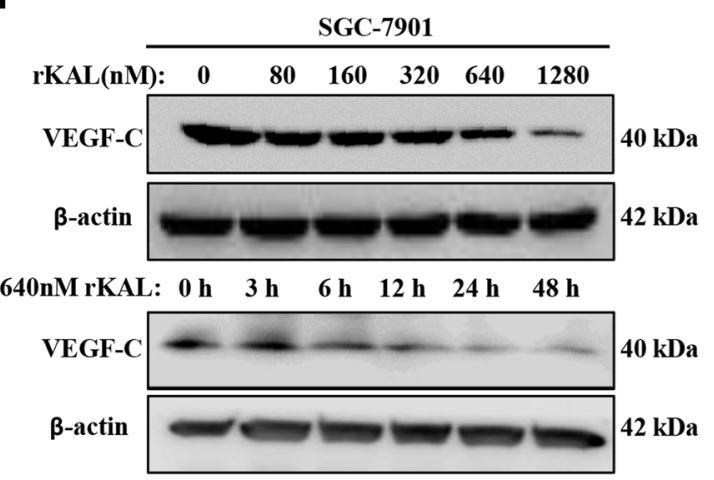

h

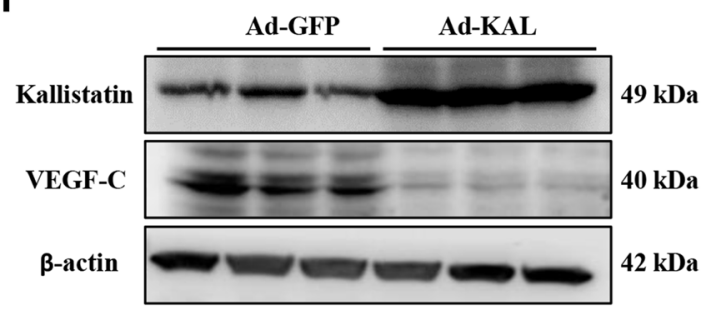

j

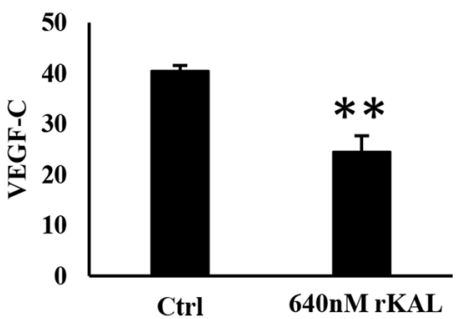

vector Ad-KAL inhibited the expression of VEGF-C in cells infected with Ad-GFP/KAL for $48 \mathrm{~h}$. Kallistatin and VEGF-C protein levels in cell lysates were measured by Western blot with $\beta$-actin as control. i, $\mathbf{j}$ Expression of VEGF-C in foot-pad gastric tumors treated with PBS or kallistatin detected by IHC. Histogram represents the expression level of VEGF-C quantified by gray-scale scanning with Image $\mathbf{J}$ in rKAL group (5) and PBS group (5). Statistical significance determined with $t$ test at $* p<0.05,{ }^{* *} p<0.01$. Results are means \pm SD

signaling pathway, which regulates the transcription of VEGF-C. 
a

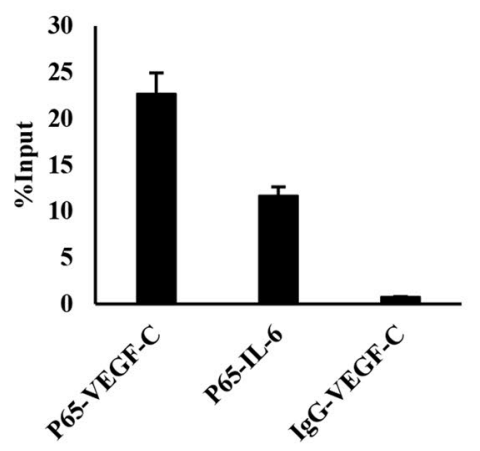

C
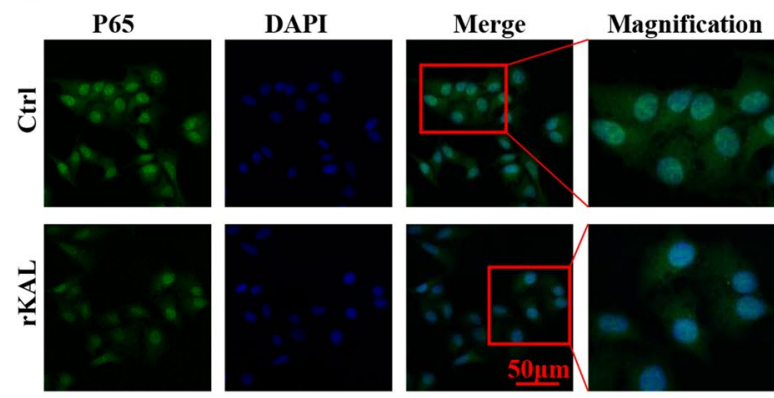

d

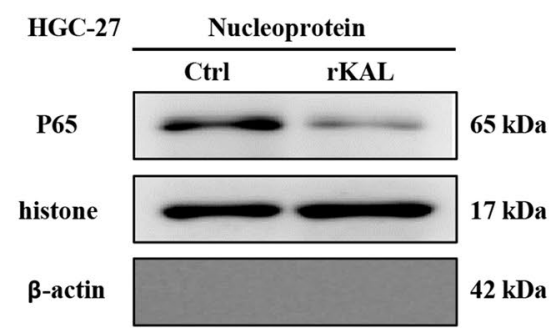

$\mathbf{f}$

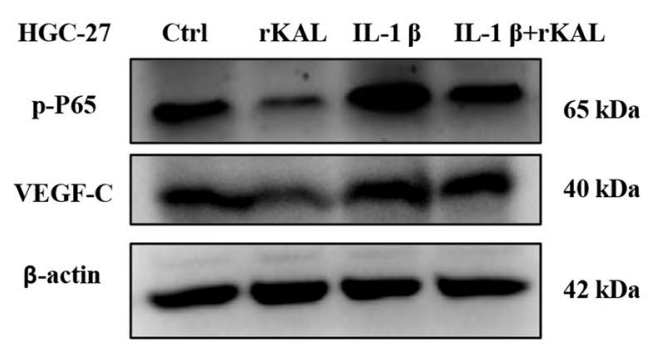

b

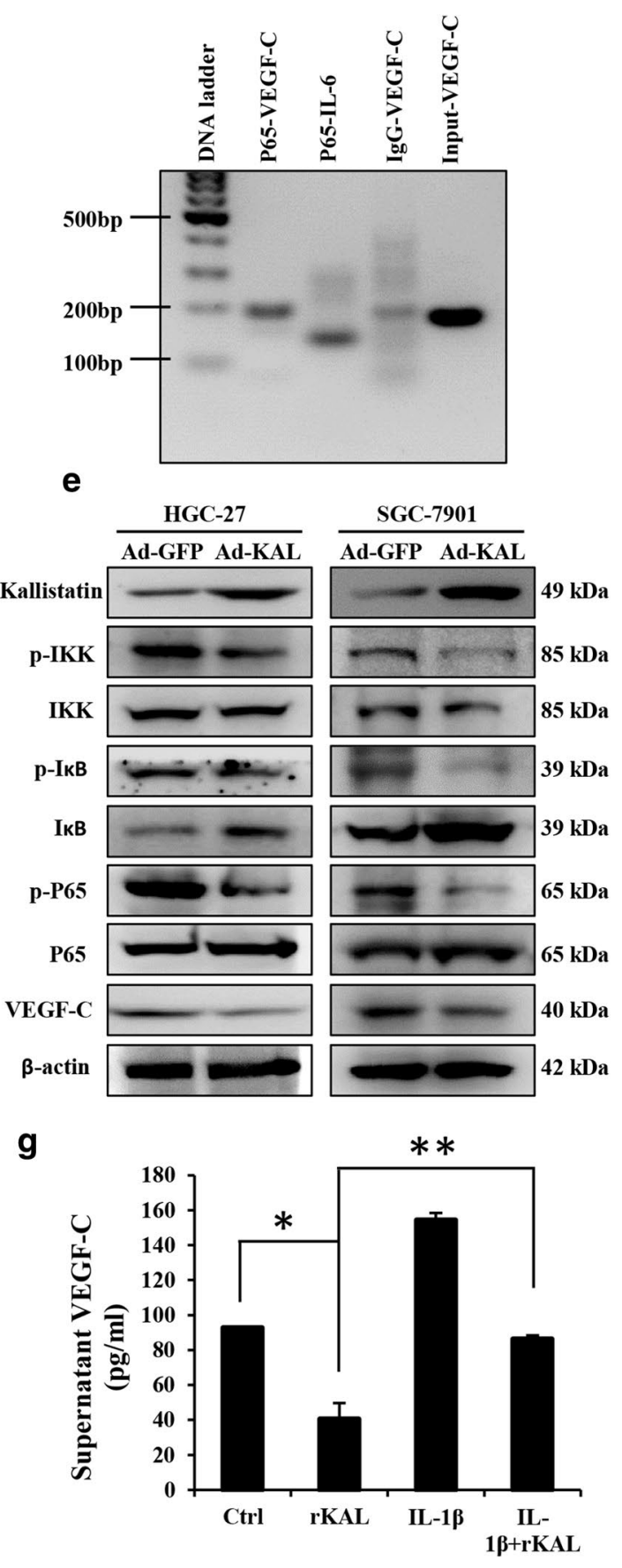

Activation of NF-кB signaling pathway in HGC-27 after kallistatin treatment: IKK/p-IKK, ІкB/p-IкB, P65/p-P65, and VEGF-C protein levels in gastric cancer cell lysates were measured by Western blot. f, g Downregulated VEGF-C level was rescued by NF-кB activator IL-1 $\beta(0.1 \mathrm{ng} / \mathrm{ml})$. Supernatant VEGF-C was detected with an ELISA kit and protein level was measured by Western blot. Results are mean \pm SD. Statistical significance was determined by one-way analysis of variance (ANOVA): $* p<0.05, * * p<0.01$ 
a

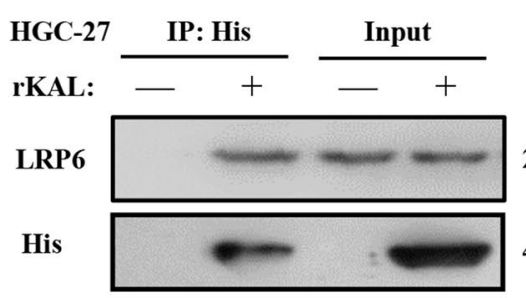

$210 \mathrm{kDa}$

$49 \mathrm{kDa}$

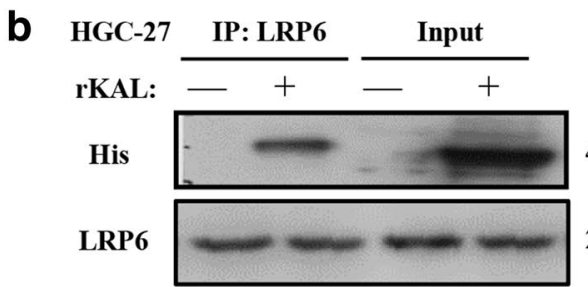

$49 \mathrm{kDa}$

$210 \mathrm{kDa}$

d

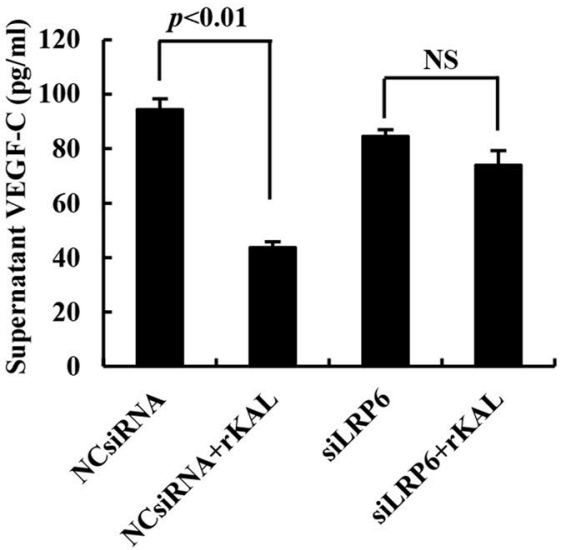

C

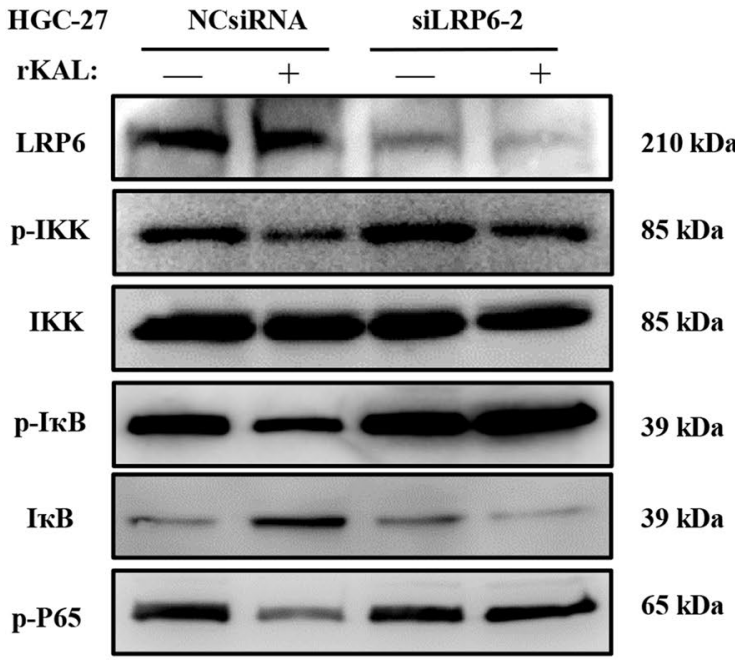

P65

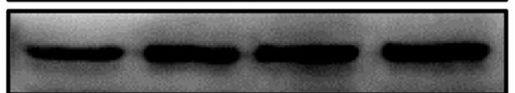

$65 \mathrm{kDa}$

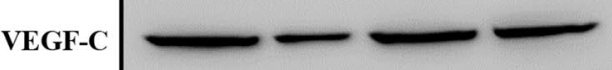

$40 \mathrm{kDa}$

$\beta$-actin

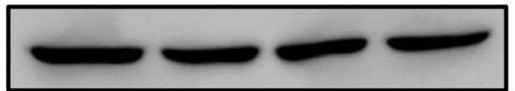

$42 \mathrm{kDa}$

e

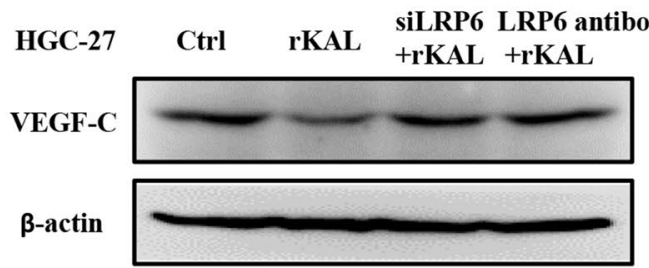

$40 \mathrm{kDa}$

$42 \mathrm{kDa}$

$\mathbf{f}$

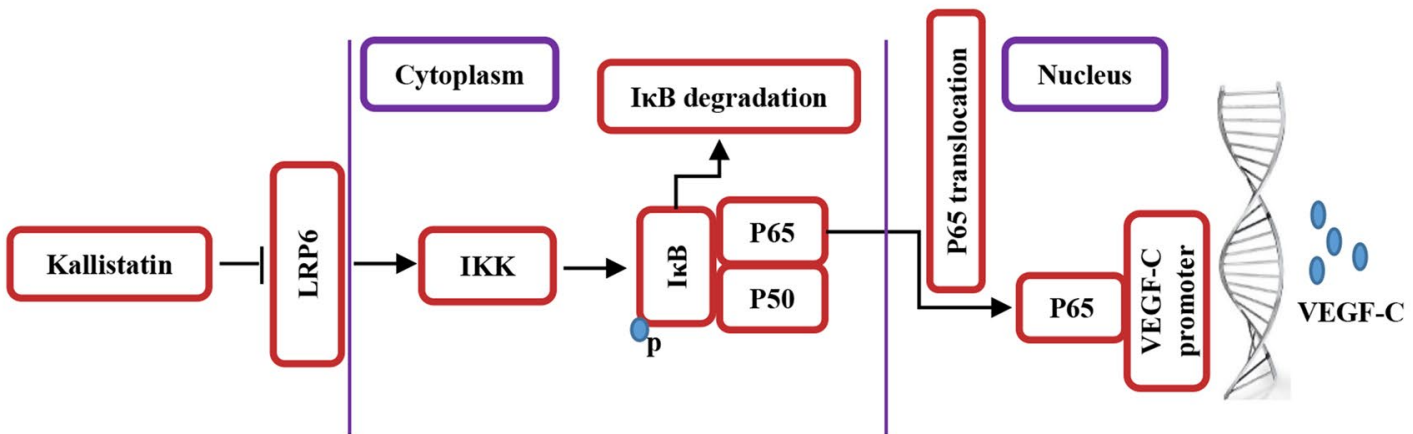

Fig. 6 Kallistatin inhibited the activation of the NF-кВ signal pathway and the expression and secretion of VEGF-C through LRP6. a, b Binding activity of kallistatin and LRP6 was detected by Co-IP with HGC-27. c, d Effects of kallistatin on NF- $\mathrm{kB}$ activity and VEGF$\mathrm{C}$ expression/secretion were blocked after knockdown of LRP6. VEGF-C protein levels in cell lysates were measured by Western blot; supernatant VEGF-C was detected by an ELISA kit. e The regulatory effect of kallistatin on VEGF-C expression was blocked after LRP6 was neutralized by its antibody. f Schematic diagram shows the mechanism of kallistatin downregulating the expression and secretion of VEGF-C in gastric cancer cells. Statistical significance was determined using the $t$ test. Results are mean \pm SD. NS nonsignificant 


\section{LRP6 served as a receptor to induce the inhibitory effect of kallistatin}

Our previous study verified that kallistatin binds to LRP6 with a high specificity and affinity, and that LRP6 can assist its inhibitory function on angiogenesis [24]. To investigate the receptor of kallistatin in gastric cancer cells, we conducted a co-IP with kallistatin and LRP6. Our results revealed that kallistatin can bind to LRP6 with a high affinity in gastric cancer cells HGC-27 (Fig. 6a, b). When LRP6 was knocked down and neutralized with siRNA and antibody, changes in NF-кВ activation, VEGF-C expression, and secretion in gastric cancer cells were detected. Consequently, the inhibitory effect on NF-KB and VEGF-C was blocked, suggesting that kallistatin downregulated VEGF-C expression and secretion through LRP6 (Fig. 6c, d, e).

\section{Discussion}

Kallistatin (also known as kallikrein-binding protein, KBP) belongs to the serine proteinase inhibitor (serpin) superfamily. Similar to other serpins, such as pigment epitheliumderived factor (PEDF), kallistatin can inhibit angiogenesis [24]. Moreover, kallistatin participates in a series of pathophysiological processes such as hypertension, inflammation, diabetes mellitus, and tumor progression [21, 24-26]. Ma et al. showed that kallistatin effectively inhibited retinal neovascularization and decreased vascular leakage in an oxygen-induced retinopathy (OIR) model [27]. Also, kallistatin inhibited the growth of liver and stomach xenograft tumors by anti-angiogenesis in nude mice [21, 28]. Recent studies revealed the multifunctional effect of kallistatin on various kinds of tumors. Sun et al. reported lower expression of kallistatin in colorectal cancer tissues [20]. Here we, for the first time, verify that kallistatin was decreased not only in the gastric cancer tissue but also in the plasma from gastric cancer patients. These findings suggest that kallistatin is a potential indicator of gastric cancer.

Lymph node metastasis is the predominant path to distant metastasis for gastric cancer, and lymphangiogenesis is an important step in the progression of lymph node metastasis. Our findings revealed a negative relationship between plasma kallistatin and lymph node metastasis in gastric cancer. Also, the lymphangiogenesis indicator LVD in gastric cancer tissues was higher than in adjacent normal mucosa. Also, we found that plasma VEGF-C in gastric cancer patients was higher than in normal persons, and VEGF-C expression in gastric cancer tissues was also stronger than in adjacent normal mucosa. More interesting, we found that the lower the degree the gastric cancer cells differentiated, the less kallistatin was expressed. These outcomes indicate that kallistatin is a good indicator of lymph node metastasis and the malignant degree of gastric cancer. Our deeper investigation showed that kallistatin significantly decreased the expression and secretion of VEGF-C in gastric cancer cells. Considering that VEGF-C is a potent promoter of lymph node metastasis, kallistatin could be expected to be an effective inhibitor of lymph node metastasis in gastric cancer.

The popliteal lymph node metastasis model is considered an effective model to mimic the process of lymph node metastasis in vivo [29]. We employed this model to confirm the inhibitory activity of kallistatin on lymph node metastasis of gastric cancer. We found that kallistatin significantly inhibited the lymph node metastasis of gastric cancer. Taken together, both in vitro and in vivo experiments suggest that kallistatin is a likely inhibitory agent of lymph node metastasis in gastric cancer.

Nuclear factor- $\kappa \mathrm{B}(\mathrm{NF}-\kappa \mathrm{B})$ signaling influences a broad range of biological processes including innate and adaptive immunity, inflammation, stress responses, B-cell development, and lymphoid organogenesis [30]. Previous studies reported that the NF- $\mathrm{kB}$ signaling pathway could be inhibited by kallistatin, and that it was involved in the regulation of VEGF-C expression in cancer cells [22, 31]. NF-kB P65 is a multifunctional transcription factor regulating a series of genes [32]. Our research clarifies that kallistatin reduced the activation of IKK, and the phosphorylation of IKB, which led to the degradation of I $\kappa \mathrm{B}$. Finally, the weakened translocation of P65 became the cause of the downregulated VEGF-C expression.

LRP6 is a single-pass transmembrane protein belonging to the low-density lipoprotein receptor (LDLR)-related protein family [33]. It functions as co-receptor for Wnt and is required for the canonical $\mathrm{Wnt} / \beta$-catenin signaling pathway [34]. Upon stimulation with Wnt, LRP6 is phosphorylated at multiple sites including Thr1479, Ser1490, and Thr1493 by kinases such as GSK-3 and CK1. Phosphorylated LRP6 recruits axin to the membrane and presumably activates $\beta$-catenin signaling $[35,36]$. Previous research verified that LRP6 could bind with kallistatin and induced its biological activity [37]. More, LRP6 was reported to be expressed in such kinds of cancer cells as breast cancer cells, pancreatic cancer cells, and gastric cancer cells [38-42], which prompted us to test whether LRP6 is involved in the inhibition of the NF- $\mathrm{KB}$ signaling pathway by kallistatin. The results showed that when we knockdown or neutralize LRP6, the activity of kallistatin is influenced, in turn suggesting that LRP6 acted as a receptor of kallistatin to induce its inhibitory effect on VEGF-C expression.

As an important member of Serpins, kallistatin has been reported to have various biological activities including its effects on tumors, such as inhibiting tumorinduced angiogenesis [21]. For this reason, kallistatin is expected to function in blocking the blood vessel metastasis of cancer cells. Kallistatin is an endogenous protein 
expressed in various organs [42], and we found its abnormal decrease in the cancer tissues and plasma of gastric cancer patients, indicating that kallistatin deficiency might be an important cause of gastric cancer. Furthermore, we revealed that kallistatin expression was related to differentiation of gastric cancer cell lines, which suggested that the decrease in kallistatin might enhance the malignancy of gastric cancer cells. These findings suggest kallistatin is a potential indicator of the development and prognosis of gastric cancer. Lymph node metastasis is the predominant way to metastasize for gastric cancer, so preventing lymph node metastasis is an important step during the treatment of gastric cancer patients. We verified the activity of kallistatin on inhibiting lymphatic metastasis of gastric cancer and clarified the molecular mechanism (Fig. 6f). Compared with other angiogenesis inhibitors, kallistatin holds the advantage that it is effective in inhibiting both angiogenesis and lymphangiogenesis. Considering that both blood vessel metastasis and lymph node metastasis occur in gastric cancer, kallistatin may be a promising candidate medicine for the treatment of gastric cancer.

In conclusion, kallistatin is a significant protein with prospects for application in gastric cancer, with weak side effects to gastric cancer patients; the potential to be a biomarker for the diagnosis of gastric cancer progression; and dual therapeutic effects on gastric cancer by inhibiting both angiogenesis and lymphangiogenesis.

Acknowledgements This study was supported by National Nature Science Foundation of China, Grant Numbers: 81572342 , 81770808, 81600641, 81471033, 81370945, 81400639, 81570871, 81570764; National Key Sci-Tech Special Project of China, Grant Numbers: 2013ZX09102053, 2015GKS355. Program for Doctoral Station in University, Grant Number: 20130171110053; Key Project of Nature Science Foundation of Guangdong Province, China, Grant Numbers: 2015A030311043, 2016A030311035. Guandong Natural Science Fund, Grant Numbers: 2014A030313073, 2015A030313103, 2015A030313029. Guandong Science and Technology Project (2014A020212023, 2015B090903063, 2017A020215075); Key Sci-tech Research Project of Guangzhou Municipality, China, Grant Numbers: 2014J4100162, 201508020033, 2016A020214001, 201607010200, 201707010084; Changjiang Scholars and Innovative Research Team in University, number 985, project PCSIRT 0947; Fundamental Research Funds for the Central Universities of China (youth program 13ykpy06, 14ykpy05, 16ykpy24). 2017 Milstein Medical Asian American Partnership Foundation Research Project Award in Translational Medicine. The funding agencies had no role in study design, data collection, and analysis; decision to publish; or preparation of the manuscript.

Author contributions X.Y. and G.G. designed the experiments and revised the manuscript; C.M., C.L., and H.Y. carried out the majority of the experiments and analyzed the data; C.M. organized the figures and wrote the manuscript; others participated in the experiments. All authors read and approved the final manuscript.

\section{Compliance with ethical standards}

Conflict of interest The authors declare that they have no conflict of interest, including relevant financial interests, activities, relationships, or affiliations.

Ethical standards All procedures followed were in accordance with the ethical standards of the responsible committee on human experimentation (institutional and national) and with the Helsinki Declaration of 1964 and later versions. Informed consent or a substitute for it was obtained from all patients included in the study.

\section{References}

1. Guggenheim DE, Shah MA. Gastric cancer epidemiology and risk factors. J Surg Oncol. 2013;107(3):230-6.

2. Siegel RL, Miller KD, Jemal A. Cancer statistics, 2017. CA Cancer J Clin. 2017;67(1):7-30.

3. Tammela T, Enholm B, Alitalo K, Paavonen K. The biology of vascular endothelial growth factors. Cardiovasc Res. 2005;65(3):550-63.

4. Yonemura Y, Endo Y, Tabata K, Kawamura T, Yun HY, Bandou $\mathrm{E}$, et al. Role of VEGF-C and VEGF-D in lymphangiogenesis in gastric cancer. Int J Clin Oncol. 2005;10(5):318-27.

5. Tammela T, Alitalo K. Lymphangiogenesis: molecular mechanisms and future promise. Cell. 2010;140(4):460-76.

6. Paduch R. The role of lymphangiogenesis and angiogenesis in tumor metastasis. Cell Oncol (Dordr). 2016;39(5):397-410.

7. Scavelli C, Vacca A, Di Pietro G, Dammacco F, Ribatti D. Crosstalk between angiogenesis and lymphangiogenesis in tumor progression. Leukemia. 2004;18(6):1054-8.

8. Streit M, Detmar M. Angiogenesis, lymphangiogenesis, and melanoma metastasis. Oncogene. 2003;22(20):3172-9.

9. Zhang L, Zhou F, Han W, Shen B, Luo J, Shibuya M, et al. VEGFR-3 ligand-binding and kinase activity are required for lymphangiogenesis but not for angiogenesis. Cell Res. 2010;20(12):1319-31.

10. Karkkainen MJ, Haiko P, Sainio K, Partanen J, Taipale J, Petrova $\mathrm{TV}$, et al. Vascular endothelial growth factor $\mathrm{C}$ is required for sprouting of the first lymphatic vessels from embryonic veins. Nat Immunol. 2004;5(1):74-80.

11. Zhao YC, Ni XJ, Wang MH, Zha XM, Zhao Y, Wang S. Tumorderived VEGF-C, but not VEGF-D, promotes sentinel lymph node lymphangiogenesis prior to metastasis in breast cancer patients. Med Oncol. 2012;29(4):2594-600.

12. Chai KX, Chen LM, Chao J, Chao L. Kallistatin: a novel human serine proteinase inhibitor. Molecular cloning, tissue distribution, and expression in Escherichia coli. J Biol Chem. 1993;268(32):24498-505.

13. Miao RQ, Agata J, Chao L, Chao J. Kallistatin is a new inhibitor of angiogenesis and tumor growth. Blood. 2002;100(9):3245-52.

14. Lu SL, Tsai CY, Luo YH, Kuo CF, Lin WC, Chang YT, et al. Kallistatin modulates immune cells and confers anti-inflammatory response to protect mice from group A streptococcal infection. Antimicrob Agents Chemother. 2013;57(11):5366-72.

15. Yin H, Gao L, Shen B, Chao L, Chao J. Kallistatin inhibits vascular inflammation by antagonizing tumor necrosis factoralpha-induced nuclear factor kappaB activation. Hypertension. 2010;56(2):260-7.

16. Chao J, Stallone JN, Liang YM, Chen LM, Wang DZ, Chao L. Kallistatin is a potent new vasodilator. J Clin Invest. 1997;100(1):11-7. 
17. Tse LY, Sun X, Jiang H, Dong X, Fung PW, Farzaneh F, et al. Adeno-associated virus-mediated expression of kallistatin suppresses local and remote hepatocellular carcinomas. J Gene Med. 2008;10(5):508-17.

18. Diao Y, Ma J, Xiao WD, Luo J, Li XY, Chu KW, et al. Inhibition of angiogenesis and HCT-116 xenograft tumor growth in mice by kallistatin. World J Gastroenterol. 2007;13(34):4615-9.

19. Ma C, Yin H, Zhong J, Zhang Y, Luo C, Che D, et al. Kallistatin exerts anti-lymphangiogenic effects by inhibiting lymphatic endothelial cell proliferation, migration and tube formation. Int $\mathbf{J}$ Oncol. 2017;50(6):2000-10.

20. Sun HM, Mi YS, Yu FD, Han Y, Liu XS, Lu S, et al. SERPINA4 is a novel independent prognostic indicator and a potential therapeutic target for colorectal cancer. Am J Cancer Res. 2016;6(8):1636-49.

21. Zhu B, Lu L, Cai W, Yang X, Li C, Yang Z, et al. Kallikreinbinding protein inhibits growth of gastric carcinoma by reducing vascular endothelial growth factor production and angiogenesis. Mol Cancer Ther. 2007;6(12 pt 1):3297-306.

22. Lin C, Song L, Gong H, Liu A, Lin X, Wu J, et al. Nkx2-8 downregulation promotes angiogenesis and activates NF-kappaB in esophageal cancer. Cancer Res. 2013;73(12):3638-48.

23. Huang KF, Huang XP, Xiao GQ, Yang HY, Lin JS, Diao Y. Kallistatin, a novel anti-angiogenesis agent, inhibits angiogenesis via inhibition of the NF-kappaB signaling pathway. Biomed Pharmacother. 2014;68(4):455-61.

24. Liu X, Zhang B, McBride JD, Zhou K, Lee K, Zhou Y, et al. Antiangiogenic and antineuroinflammatory effects of kallistatin through interactions with the canonical Wnt pathway. Diabetes. 2013;62(12):4228-38.

25. Chao J, Chao L. Kallistatin in blood pressure regulation transgenic and somatic gene delivery studies. Trends Cardiovasc Med. 1997;7(8):307-11.

26. Jenkins AJ, McBride JD, Januszewski AS, Karschimkus CS, Zhang B, O'Neal DN, et al. Increased serum kallistatin levels in type 1 diabetes patients with vascular complications. J Angiogenes Res. 2010;2:19.

27. Xu Y, Zhao H, Zheng Y, Gu Q, Ma J, Xu X. A novel antiangiogenic peptide derived from hepatocyte growth factor inhibits neovascularization in vitro and in vivo. Mol Vis. 2010;16:1982-95.

28. Jiang X, Li H, Qiao H, Jiang H, Xu R, Sun X. Combining kallistatin gene therapy and meloxicam to treat hepatocellular carcinoma in mice. Cancer Sci. 2009;100(11):2226-33.

29. Liu L, Lin C, Liang W, Wu S, Liu A, Wu J, et al. TBL1XR1 promotes lymphangiogenesis and lymphatic metastasis in esophageal squamous cell carcinoma. Gut. 2015;64(1):26-36.
30. Hayden MS, Ghosh S. NF-kappaB, the first quarter-century: remarkable progress and outstanding questions. Genes Dev. 2012;26(3):203-34.

31. Wang T, Shi F, Wang J, Liu Z, Su J. Kallistatin suppresses cell proliferation, invasion, and promotes apoptosis in cervical cancer through blocking NF-kappaB signaling. Oncol Res. 2016. https:// doi.org/10.3727/096504016X14799180778233.

32. Oeckinghaus A, Hayden MS, Ghosh S. Crosstalk in NF-kappaB signaling pathways. Nat Immunol. 2011;12(8):695-708.

33. Brown SD, Twells RC, Hey PJ, Cox RD, Levy ER, Soderman AR, et al. Isolation and characterization of LRP6, a novel member of the low density lipoprotein receptor gene family. Biochem Biophys Res Commun. 1998;248(3):879-88.

34. Tamai K, Semenov M, Kato Y, Spokony R, Liu C, Katsuyama Y, et al. LDL-receptor-related proteins in Wnt signal transduction. Nature. 2000;407(6803):530-5.

35. Acebron SP, Niehrs C. Beta-catenin-independent roles of Wnt/ lrp6 signaling. Trends Cell Biol. 2016;26(12):956-67.

36. Perrody E, Abrami L, Feldman M, Kunz B, Urbe S, van der Goot FG. Ubiquitin-dependent folding of the Wnt signaling coreceptor LRP6. Elife. 2016. https://doi.org/10.7554/eLife.19083 (eng).

37. Zhang J, Yang Z, Li P, Bledsoe G, Chao L, Chao J. Kallistatin antagonizes $\mathrm{Wnt} /$ beta-catenin signaling and cancer cell motility via binding to low-density lipoprotein receptor-related protein 6 . Mol Cell Biochem. 2013;379(1-2):295-301.

38. Hong Y, Manoharan I, Suryawanshi A, Shanmugam A, Swafford D, Ahmad S, et al. Deletion of LRP5 and LRP6 in dendritic cells enhances antitumor immunity. Oncoimmunology. 2016;5(4):e1115941.

39. Garg B, Giri B, Majumder K, Dudeja V, Banerjee S, Saluja A. Modulation of post-translational modifications in beta-catenin and LRP6 inhibits Wnt signaling pathway in pancreatic cancer. Cancer Lett. 2016;388:64-72.

40. Wang Z, Li B, Zhou L, Yu S, Su Z, Song J, et al. Prodigiosin inhibits Wnt/beta-catenin signaling and exerts anticancer activity in breast cancer cells. Proc Natl Acad Sci USA. 2016;113(46):13150-5.

41. Zheng R, Deng Q, Liu Y, Zhao P. Curcumin inhibits gastric carcinoma cell growth and induces apoptosis by suppressing the Wnt/ beta-catenin signaling pathway. Med Sci Monit. 2017;23:163-71.

42. Chao J, Schmaier A, Chen LM, Yang Z, Chao L. Kallistatin, a novel human tissue kallikrein inhibitor: levels in body fluids, blood cells, and tissues in health and disease. J Lab Clin Med. 1996;127(6):612-20. 